\title{
Addendum
}

\section{Representations of quantum algebras}

\author{
H.H. Andersen, P. Polo, K. Wen
}

Matematisk Institut, Aarhus Universitet, Ny Munkegade, DK-8000 Aarhus C, Denmark

Invent. math. 104, 1-59 (1991)

It has been pointed out to us (see the Introduction of [1]) that in Section 5 of the above paper we are implicitly using a non-obvious Mackey type result when we apply the results of Section 4. In [1], Lin proves in a more general context that one does indeed have such Mackey properties, at least over a base field (see Remark 2 below).

Here we show that the result we need follows in fact quite easily from arguments in our paper. Let the algebras $U^{b}$ and $U^{b}(i), U_{i}$, for $i=1, \ldots, n$, be defined as in our paper, see p. 4 and 2.5. (We take this opportunity to point out that, on p. 4, $U(I)$ should read $U^{\mathrm{b}}(I)$.) Let $U_{i}^{\mathrm{b}}$ denote the subalgebra of $U_{t}$ generated by $K_{i}^{ \pm 1}$ and $F_{i}^{(r)}$, for $r \geqq 0$. Then, with notation as in our paper, one has:

Proposition 5.0. Let $M \in \mathscr{C}^{\natural}$. For any $i=1, \ldots, n$ and $r \geqq 0$, one has natural isomorphisms of $U_{i}$-modules

$$
\left.H^{r}\left(U^{b}(i) / U^{b}, M\right)\right|_{U_{t}} \simeq H^{r}\left(U_{i} / U_{i}^{b},\left.M\right|_{U_{I}^{p}}\right) .
$$

Proof. For $r=0$ the statement follows directly from the definition of the induction functors in Section 2. To obtain it for all $r$ it is enough (by the usual arguments based on the standard resolutions of 2.17) to check that $H^{r}\left(U_{i} / U_{i}^{\mathrm{b}}, I(\mu)\right)=0$, for $r>0$, where $I(\mu)$ denotes $H^{0}\left(U^{\mathrm{b}} / U^{0}, \mathscr{A}_{\mu}\right)$, for $\mu \in X$. It follows from the definition that $H^{0}\left(U_{l} / U_{i}^{b},-\right)$ commutes with direct limits. Moreover, by 2.13 the category $\mathscr{C}_{i}^{b}$ has enough injectives and, using the fact that any object in $\mathscr{C}_{l}^{b}$ is a union of $\mathscr{A}$-finite $U_{i}^{b}$-submodules, one obtains that a direct limit of injective objects in $\mathscr{C}_{i}^{b}$ is injective. By standard arguments it then follows that $H^{r}\left(U_{i} / U_{i}^{b},-\right)$ commutes with direct limits, for $r \geqq 0$. But $I(\mu)$ is the direct limit of $U^{b}$-submodules $V_{m} \simeq H^{0}(m \rho) \otimes \mathscr{A}_{m \rho+\mu}$, for $m \geqq 0$, by Lemma 5.3 (note that this lemma does not use the results of 5.1-5.2) and hence, for $r \geqq 0, H^{r}\left(U_{i} / U_{i}^{b}, I(\mu)\right)$ is the direct limit of $H^{r}\left(U_{i} / U_{i}^{b}, V_{m}\right)$, for $m \rightarrow \infty$. Finally, using Corollary 3.3 (i) and Proposition 2.16, one obtains 
$H^{r}\left(U_{i} / U_{i}^{b}, V_{m}\right) \simeq H^{0}(m \rho) \otimes H^{r}\left(U_{i} / U_{i}^{b}, \mathscr{A}_{m \rho+\mu}\right)$ and, by Proposition 4.2, this vanishes for $r>0$ and $m \gg 0$. The proposition follows.

Remarks. 1. The proposition is used implicitly in 5.1, 5.4-5.8.

2. Let $I \subseteq\{1, \ldots, n\}$. Using Corollary 5.7 , applied to the algebra $U_{l}$, one then obtains that the proposition generalizes to the case of induction from $U^{b}$ to $U^{b}(I)$. Over a base field this is proved by different arguments in [1].

[1] Lin, Z.: A Mackey Decomposition Theorem and Cohomology for Quantum Groups at Roots of 1. J. Algebra 166, 100-129 (1994) 